\title{
Hábitos alimentarios del venado cola blanca Odocoileus virginianus (Artiodactyla: Cervidae) en el Parque Natural Sierra Nanchititla, Estado de México
}

\author{
Ulises Aguilera-Reyes ${ }^{1}$, Víctor Sánchez-Cordero ${ }^{2}$, José Ramírez-Pulido ${ }^{3}$, \\ Octavio Monroy-Vilchis ${ }^{1}$, Georgina Isabel García López ${ }^{1} \&$ Mariusz Janczur ${ }^{1}$ \\ 1. Centro de Investigación en Recursos Bióticos, Facultad de Ciencias, Universidad Autónoma del Estado de México. \\ Instituto Literario No. 100 Oriente, Colonia Centro, Toluca, Estado de México 50000. México; \\ uaguilera22@gmail.com \\ 2. Departamento de Zoología, Instituto de Biología, U.N.A.M. Circuito exterior s/n, Ciudad Universitaria, Copilco, \\ Coyoacán. A.P. 70-153/70-233 México, Distrito Federal. C.P. 04510; victor@ibiologia.unam.mx \\ 3. Departamento de Biología, División de C.B.S Universidad Autónoma Metropolitana. Unidad Iztapalapa, Av. San \\ Rafael Atlixco No. 186, Col. Vicentina C.P.09340 Del. Iztapalapa México D.F.; jrp@xanum.uam.mx
}

Recibido 14-XI-2011. Corregido 20-VII-2012. Aceptado 21-VIII-2012.

\begin{abstract}
Food habits of the white-tailed deer, Odocoileus virginianus (Artiodactyla: Cervidae) in Nanchititla Natural Park, Mexico. White-tailed deer is a species with a large behavioral plasticity and adaptation to different habitats, including their food habits. This study was conducted with the aim to determine the food habits of this species in the cloud (BMM) and pine-oak (BPE) forests. Deer scats and plant samples were obtained following standard methods, from Sierra Nanchititla Park in the State of Mexico, from June 1990 to May 1992. A total of 104 deer pellet-groups were collected, and histological analysis for herbivores was used and compared with stock samples of plant tissues collected from the study area. We applied the Spearman correlation and Morisita index to determine alimentary preference. The results showed that the deer consumes $79.44 \%$ of plant species from BMM and $20.56 \%$ of the BPE. There is a selectivity tendency for 12 of the 14 plant species located in the BMM, while for BPE no tendency was observed. Key species that are part of the elemental diet of the deer in these areas were: Acalypha setosa, Smilax pringlei, Psidium sartorianum and Dendropanax arborea. The consumption of plants did not differ significantly between the dry and rainy seasons in terms of biological form, however, during the dry season there is a tendency to consume trees, and by the end of the rainy season to consume herbs. The data indicate that the deer can be selective with BMM plants, while for the BPE tends to be opportunistic. Rev. Biol. Trop. 61 (1): 243-253. Epub 2013 March 01.
\end{abstract}

Key words: white-tailed deer, food habits, cloud forest, pine-oak forest, Mexico.

La distribución geográfica del venado cola blanca (Odocoileus virginianus) ocurre en la mayor parte de México, con excepción de la península de Baja California y el norte de Sonora (Hall 1981, Ceballos \& Oliva 2005). En el centro de México, habita la subespecie Odocoileus virginianus miquihuanensis (Hall 1981, Ceballos \& Oliva 2005). El conocimiento de los hábitos alimentarios del venado cola blanca en diferentes hábitat, ha permitido establecer la gran adaptación de este herbívoro en diferentes tipos de vegetación, ya que consume especies herbáceas, arbustivas y arbóreas, comportándose como una especie oportunista o selectiva según las condiciones ecológicas de los sitios en donde habita (Gallina 1978, 1981, 1993, Narváez et al. 1983, Clemente 1984, Morales-García 1985, 
Villarreal \& Gabriel 1986, McCullough 1985, Medina-Flores 1986, Di Mare 1994, Vaughan \& Rodríguez 1995, Martínez et al. 1997, ColínNegrete \& Bernal-Barragán 2005, Ortega et al. 2011, Hernández-Silva et al. 2011).

El venado puede percibir la calidad de una planta con base en su palatabilidad, consumiendo aquellas partes que son altamente digeribles y le brindan mayores nutrimentos. Para tener acceso a estas características pueden verse obligados a consumir ciertas plantas, aún con el costo que les representa la ingestión de compuestos secundarios que pueden ser tóxicos en menor o mayor medida. Por ejemplo los herbívoros, en épocas de invierno en zonas templadas o frías donde se reduce la disponibilidad de alimento, consumen aquellas plantas que les reditúan beneficio en el aporte de grasa, aun a costa de que estas plantas contengan los compuestos mencionados (Smith 1991, Provenza et al. 1992, Ernest 1994, Vangilder et al. 1982, Henke et al. 1988, SilvaVillalobos et al. 1999, Arceo et al. 2005, Plata et al. 2009). Diversas técnicas se han usado para determinar los hábitos alimentarios del venado cola blanca. Sin embargo uno de los más utilizados es a través del análisis de los restos de tejido vegetal presentes en los excrementos (Camargo-Sanabria \& Mandujano 2011, Gallina 1993, Mandujano 2011).

Los estudios sobre la ecología alimentaria del venado se han desarrollado principalmente en bosques templados, tropicales y semiáridos, en cambio, son escasos en sitios que involucran el Bosque Mesófilo de Montaña (VillarrealEspino-Barros 2008). Por lo que el presente trabajo tiene como objetivo determinar los hábitos alimentarios del venado cola blanca (Odocoileus virginianus texanus) en este tipo de bosque, y compararlo con los del bosque de pino-encino, así como establecer la posible selectividad en la alimentación entre la época seca y lluviosa y las formas biológicas de las plantas.

\section{MATERIALES Y MÉTODOS}

Área de estudio: El presente estudio se desarrolló en el Parque Natural Sierra
Nanchititla, ubicado en el Municipio de Luvianos en el Estado de México. El Parque se encuentra dividido en dos zonas: una menor de aproximadamente 20ha, donde se localizan alrededor de 20 venados cola blanca (Odocoileus virginianus texanus), y donde fueron liberados cinco venados jóvenes en junio de 1990, y una zona mayor de 1508 ha, donde se encuentra la mayor población. En esta zona, se liberaron 16 venados (Odocoileus virginianus texanus) en septiembre de 1987, y 25 más fueron liberados en agosto de 1988, para un total de 41 .

El Parque se localiza en la región Fisiográfica de la Cuenca del Balsas, en la Provincia de la Sierra Madre del Sur, Sub-provincia Depresión del Balsas (INEGI 1987). Las coordenadas de la reserva (RNSN) son, 100 $36^{\circ}$ '49'

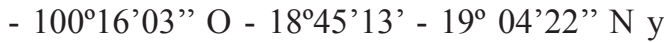
cuenta con una extensión de 1528 ha.

Clima: En las altitudes de 700 a los $2000 \mathrm{msnm}$, en la región correspondiente a la Depresión del Balsas, las precipitaciones son más abundantes en el verano. La orientación del relieve hacia el sur recibe más insolación y por ello tiene una mayor temperatura promedio que la del Norte, esto condiciona las características climáticas de esta zona, con los climas cálido Awo(w-) hacia los transicionales, cálidos templados, con las variantes más o menos húmedos $\mathrm{A}(\mathrm{C}) \mathrm{w}(\mathrm{w})$ y $(\mathrm{A}) \mathrm{C}(\mathrm{w} 2)(\mathrm{w})$, de acuerdo con la clasificación de Köppen, modificada por (García 1981), en los que se definen dos épocas la de estiaje de noviembre a abril y la de lluvias de mayo a octubre.

Vegetación: Todas las variaciones en la riqueza geomorfológica y climática del Parque Estatal dan por resultado diversos tipos de vegetación; en el presente estudio se enlistan, los dos bosques referidos para el presente estudio de acuerdo con Aguilar-Ortigoza (1994) y Zepeda-Gómez \& Velázquez-Montes (1999).

Bosque mixto de pino-encino (BPE): Corresponde a la vegetación arbórea en la que dominan diferentes especies de los géneros 
Pinus sp. y Quercus sp. En estos bosques el estrato arbóreo es el dominante y está representado principalmente por Pinus oocarpa, Quercus elliptica, Q. urbanii, Clethra mexicana, Styrax ramirezii y Arbutus xalapensis; entre las arbustivas se encuentran Salvia sessei, Tibouchinis sp. y Fuchsia microphylla; casi no existen herbáceas. En estas zonas muy perturbadas se encuentran grandes espacios abiertos y se llegan a establecer palmas del género $\mathrm{Bra}$ hea. La mayor parte del bosque presenta algún grado de perturbación por incendios, resinación de pinos y extracción de leña. Dentro de la reserva este tipo de vegetación ocupa $1225 \mathrm{ha}$, que corresponden al $82 \%$ de superficie total.

\section{Bosque mesófilo de montaña (BMM):}

Se caracteriza por sus componentes principalmente arbóreos de 15 a 20 metros de alto. Entre los árboles destacan Inga histonii, Matudea triversis, Clusia salvinii, Oreopanax arborea y Eugenia sp. Muy cerca de los ríos se presentan ejemplares de Alnus firmifolia, en claros de este bosque se encuentran estratos arbustivos y herbáceos donde abundan especies de compuestas, gramíneas, rubiáceas, melastomatáceas, selaginelas, equisetos y helechos.

Recolecta de excrementos: Se utilizó un muestreo aleatorio sistemático en la zona de estudio (Bonilla 1991). Cada 15 días se colectaron los excrementos en el campo, desde junio de 1990-mayo de 1992. Se realizaron transectos fijos de $500 \mathrm{~m}$ de longitud en los dos tipos de vegetación. Los grupos fecales se agruparon de acuerdo con su fecha de colecta, sólo se colectaron aquellos excrementos que se encontraban frescos los cuales fueron en total 104 grupos fecales, 52 en cada tipo de vegetación de los cuales 27 correspondieron a la época de estiaje y 27 a la de lluvia.

Muestreo de la vegetación: Se realizaron muestreos de vegetación en las zonas mejor conservadas del Bosque de Pino-Encino (BPE) y del Bosque Mesófilo de Montaña (BMM). El muestreo se realizó por formas biológicas esto es: estrato herbáceo, arbustivo, arbóreo y lianas. Las variables implícitas fueron; dominancia (ya sea en forma de cobertura o área basal), densidad y frecuencia para obtener el valor de importancia (VI). Estos datos permitieron establecer una relación de selectividad de las zonas donde la especie acostumbra ramonear (Brower 1977). En relación con lo anterior, se utilizó un método distinto para cada uno de los estratos. Para el estrato arbóreo se trazaron cinco líneas Canfield de $100 \mathrm{~m}$, tanto en el BPE como en el BMM, cada 20m, con el método de cuadrantes centrados en un punto; de cada una de las especies se obtuvo su VI (Morisita 1959, Cottam \& Curtis 1957, Catana 1963). Para el estrato arbustivo, sobre la misma línea se hicieron cinco cuadrantes de $64 \mathrm{~m}^{2}$ y se obtuvo también su VI. Para el estrato herbáceo se procedió de la misma forma que en el estrato arbustivo, pero utilizando cuadrantes de $1 \mathrm{~m}^{2}$, debido a las características del mismo, y se obtuvieron también los VI (Brower 1977). Las plantas presentes en estas áreas de muestreo se colectaron a fin de determinarlas y practicarles la técnica histológica. Las plantas colectadas fueron determinadas por especialistas de la Facultad de Ciencias de la Universidad Autónoma del Estado de México y depositadas en el herbario de referencia de la misma.

Para demostrar si las especies de plantas son semejantes en los dos tipos de bosques muestreados se aplicó el Índice de Similitud de Morisita (Horn 1966).

Determinación de la alimentación: $\mathrm{La}$ determinación de la composición de la alimentación del venado cola blanca, se basó en la técnica histológica para herbívoros propuesta por Holechek et al. (1982), Peña \& Habib (1980). Para realizar las observaciones de las muestras de los excrementos en el microscopio, se hicieron cinco preparaciones histológicas permanentes para cada muestra colectada. El trabajo se repitió cada 15 días y durante dos años. Las observaciones consistieron en revisar y registrar las especies de plantas encontradas en 20 campos por preparación, con un microscopio binocular Zeiss y con objetivos de $40 \mathrm{X}$ y oculares de $10 \mathrm{X}$, obteniendo 400 aumentos. 
Se acumularon un total de 100 observaciones por muestra. La identificación de las plantas presentes en las preparaciones se efectúo reconociendo características histológicas como: la forma y tamaño de pelos epidérmicos, forma de células epidérmicas y células silíceas, formas de estomas y tricomas, entre otras. Se hicieron preparaciones de plantas (hojas, ramas, frutos y flores) colectadas en los muestreos de los dos tipos de vegetación (Quintanilla et al. 1988). El porcentaje de frecuencia por especie se obtuvo por el número de campos donde apareciera la planta en un total de 100 campos por muestra (Fracker \& Brischle 1944).

Observaciones directas preliminares con venados jóvenes: Con la intención de determinar si la técnica histológica para herbívoros era adecuada, se introdujeron cinco venados jóvenes (Odocoileus virginianus texanus) en la zona de menor extensión del Parque. Estos venados fueron separados de su madre desde la primera semana de nacidos y alimentados en forma artificial por tres de los autores del presente trabajo hasta los cinco meses de edad cuando fueron liberados, se observó sus hábitos alimentarios y se determinó las especies que consumieron. Las observaciones se realizaron por la mañana y por la tarde a lo largo de dos meses. Las plantas consumidas fueron posteriormente verificadas, al colectarse los excrementos y aplicarles la técnica histológica para herbívoros. El porcentaje de eficacia de la técnica histológica aplicada a los venados jóvenes fue del $88.8 \%$, es decir de las 18 especies que consumieron y fueron determinadas por observación directa, 16 fueron correctamente identificadas a través de la técnica histológica.

Determinación de la preferencia alimentaria: Para comprobar si existen preferencias en el consumo de especies de plantas en el BMM, con respecto al BPE, se realizó una sumatoria entre los porcentajes de consumo de alimento para cada tipo de vegetación y se compararon.

Para comprobar si el venado cola blanca es selectivo en la alimentación de especies de plantas en cada tipo de vegetación, se aplicó un análisis de correlación de rangos de Spearman entre los porcentajes de consumo y los valores de importancia (VI) de las especies consumidas, se trabajó con una ( $\mathrm{p}=0.05)$ (Zar 1984).

Para verificar si existen diferencias en el consumo de plantas entre la época seca y lluviosa, se compararon los porcentajes de consumo de las formas biológicas de las plantas en ambas épocas, utilizando la prueba de Wald-Wolfowitz, por último, para comprobar la preferencia de plantas en función de su forma biológica, se aplicó una $\mathrm{X}^{2}$ de bondad de ajuste (Zar 1984).

\section{RESULTADOS}

El Índice de Similitud de Morisita aplicado a la vegetación permitió establecer que las comunidades vegetales son diferentes florísticamente, en los dos tipos de bosque muestreados, dado que el índice obtenido fue de: $1 \mathrm{M}=0.35$, lo que indicó un valor muy bajo de especies de plantas similares dada su frecuencia y cobertura (Horn 1966 citado por Krebs 1989).

En la vegetación del BMM, destaca un pasto de la familia Poaceae (Panicum $s p$ ) con (12.3 de VI), para el caso de las herbáceas, en cuanto al estrato arbustivo: Psidium guajava fue la única planta que consumió el venado con (3.0 de VI); mientras que de las especies arbóreas, destaca Psidium sartorianum con (73.5 de VI) (Cuadro 1). Con respecto al Bosque de Pino-Encino (BPE), se encontró que una sola especie fue la consumida por el venado en el estrato herbáceo: Vaccinium geminiflorum teniendo un VI de 22.2; para el estrato arbustivo: Gliciridium sepium fue la que obtuvo el VI mayor con un (38.9), finalmente de la vegetación arbórea, dos encinos: Quercus sp. y Quercus rugosa obtuvieron valores de importancia de 21 y 64 respectivamente (Cuadro 1).

De las especies de plantas que formaron parte de la alimentación de los venados se observa que tres especies fueron preferidas para su consumo en el BMM: Acalypha setosa con $(18.02 \%)$, Smilax pringlei con (16.91\%) 
CUADRO 1

Porcentaje de especies de plantas que consume el venado cola blanca en el Bosque Mesófilo de Montaña (BMM) y en el Bosque de Pino Encino (BPE)

TABLE 1

Percentage of plant species consumed by white-tailed deer in the cloud forest (BMM) and Pine-Oak Forest (BPE)

\begin{tabular}{|c|c|c|c|c|c|c|c|}
\hline \multirow[b]{2}{*}{ Familia } & \multirow[b]{2}{*}{ Especie } & \multicolumn{2}{|c|}{ Época } & \multirow[b]{2}{*}{$\begin{array}{c}\text { Total } \\
\text { consumido \% }\end{array}$} & \multicolumn{2}{|l|}{$300 \%$} & \multirow[b]{2}{*}{$\begin{array}{c}\text { Tipo de } \\
\text { vegetación }\end{array}$} \\
\hline & & $\begin{array}{c}\text { Seca } \\
\%\end{array}$ & $\begin{array}{c}\text { Lluvia } \\
\%\end{array}$ & & VI & Estratos & \\
\hline Euphorbiaceae & Acalypha setosa & 8.5 & 9.52 & 18.02 & 5 & Herbáceo & BMM \\
\hline Smilacaceae & Smilax pringlei & 9.2 & 7.71 & 16.91 & & Enredadera & BMM \\
\hline Myrtaceae & Psidium sartorianum & 1.5 & 10.21 & 11.71 & 73.5 & Arbóreo & BMM \\
\hline Araliaceae & Dendropanax arborea & 4.5 & 2.45 & 6.95 & 47 & Arbóreo & BMM \\
\hline Moraceae & Dorstenia drakeana & 2.3 & 3.31 & 5.61 & 1.8 & Herbáceo & BMM \\
\hline Clethraceae & Clethra mexicana & 1.6 & 3.71 & 5.31 & 10.8 & Arbóreo & $\mathrm{BPE}$ \\
\hline Poaceae & Panicum sp. & 4.1 & 0.80 & 4.9 & 12.3 & Herbáceo & BMM \\
\hline Melastomataceae & Asissanthera cuadrata & 0.6 & 3.58 & 4.18 & $\mathrm{~S} / \mathrm{D}$ & Herbáceo & $\mathrm{BPE}$ \\
\hline Poace & Festuca sp. & 0.7 & 2.34 & 3.04 & 0.9 & Herbáceo & BMM \\
\hline Cletraceae & Clethra aleoides & 1.8 & 0.76 & 2.56 & 17.1 & Arbóreo & BPE \\
\hline Myrtaceae & Psidium guajava & 1.4 & 1.05 & 2.45 & 3 & Arbustivo & BMM \\
\hline Clusiaceae & Clusia salvinii & 0.4 & 2.04 & 2.44 & 1.8 & Herbácea & BMM \\
\hline Oxalidaceae & Oxalis sp. & 0.00 & 2.12 & 2.12 & 1.0 & Herbáceo & BMM \\
\hline Cheranthaceae & Hedyosmum mexicanum & 0.01 & 2.07 & 2.08 & $\mathrm{~S} / \mathrm{D}$ & Arbustivo & BPE \\
\hline Euphorbiacea & Euphorbia sp. & 0.00 & 1.80 & 1.8 & $\mathrm{~S} / \mathrm{D}$ & & BMM \\
\hline Commelinaceae & Commelina sp. & 1.6 & 0.00 & 1.6 & 2 & Herbáceo & BPE \\
\hline Begonaceae & Begonia crassicaulis & 0.4 & 1.22 & 1.62 & 2.2 & Herbáceo & BMM \\
\hline Fabaceae & Gliciridium sepium & 1.5 & 0.00 & 1.5 & 38.9 & Arbustivo & BPE \\
\hline Ericaceae & Vaccinium geminiflorum & 0.00 & 1.46 & 1.46 & 22.2 & Herbáceo & BPE \\
\hline Fagaceae & Quercus rugosa & 0.9 & 0.44 & 1.34 & 64 & Arbóreo & BPE \\
\hline Mimosaceae & Inga micheliana & 1.2 & 0.00 & 1.2 & 5.7 & Arbóreo & BMM \\
\hline Lamiaceae & Salvia sp. & 0.00 & 0.65 & 0.65 & & & BMM \\
\hline Fagaceae & Quercus sp. & 0.09 & 0.44 & 0.53 & 21 & Arbóreo & BPE \\
\hline Poaceae & Mulhenbergia minutissima & 0.00 & 0.02 & 0.02 & 0.9 & Herbáceo & BMM \\
\hline TOTAL & & 42.3 & 57.7 & 100.0 & & & \\
\hline
\end{tabular}

y Psidium sartorianum con (11.71\%), además de otras once especies que tuvieron porcentajes de consumo menores (Cuadro 1); en el $\mathrm{BPE}$, destacan cuatro especies como las más consumidas por los venados: Clethra mexicana, con $5.31 \%$, Asissanthera cuadrata $4.18 \%$, Clethra aleoides $2.56 \%$ y Hedyosmum mexicanum $2.08 \%$, el resto, es decir seis especies más, tuvieron porcentajes de consumo menores al $2 \%$ (Cuadro 1). En total 24 especies de plantas fueron consumidas por los venados, 15 del BMM y 9 del BPE, todas ellas diferentes. Esto refleja la composición distinta de cada tipo de vegetación (Cuadro 1). La sumatoria de los porcentajes de consumo de especies vegetales para cada tipo de vegetación manifiesta claramente que el $79.44 \%$ del consumo se dirigió a especies localizadas en el BMM, mientras que el $20.56 \%$ fue para las especies localizadas en el BPE (Cuadro 2).

Cuatro especies fueron consumidas por los venados en zonas perturbadas del BPE; Cuphea procumbens con el $(23.69 \%)$, Pteridium aquilinum $3.83 \%$ y dos especies más no determinadas lo que indica la movilidad de los venados en la búsqueda de recursos alimentarios. 
CUADRO 2

Plantas consumidas por el venado cola blanca en dos tipos de vegetación

TABLE 2

Plants consumed by white-tailed deer in two vegetation types

\begin{tabular}{lccc}
\multicolumn{1}{c}{ Bosque Mesófilo de Montaña } & \multicolumn{2}{c}{ Bosque de Pino-Encino } \\
\multicolumn{1}{c}{ Especie } & E Total consumido & \% Total consumido \\
Acalypha setosa & 18.02 & Clethra mexicana & 5.31 \\
Smilax pringlei & 16.91 & Asissanthera cuadrata & 4.18 \\
Psidium sartorianum & 11.71 & Clethra aleoides & 2.56 \\
Dendropanax arborea & 6.95 & Hedyosmum mexicanum & 2.08 \\
Dorstenia drakeana & 5.61 & Commelina sp. & 1.6 \\
Panicum sp. & 4.9 & Gliciridium sepium & 1.5 \\
Festuca sp. & 3.04 & Vaccinium geminiflorum & 1.46 \\
Psidium guajava & 2.45 & Quercus rugosa & 0.53 \\
Clusia salvinii & 2.44 & Quercus sp. & \\
Oxalis sp. & 2.12 & & \\
Euphorbia sp. & 1.8 & & \\
Begonia crassicaulis & 1.62 & & \\
Inga micheliana & 1.2 & & \\
Salvia sp. & 0.65 & & \\
Mulhenbergia minutissima & 0.02 & & \\
TOTAL & 79.44 & & \\
\hline
\end{tabular}

La correlación de rangos de Spearman para determinar la relación entre el valor de importancia de las plantas y el porcentaje de consumo en el BMM, no muestran una relación entre las dos variables analizadas ( $\mathrm{rs}=-0.111$, $\mathrm{p}<0.05)$. Para el BPE los cálculos muestran una correlación muy baja ( $\mathrm{rs}=0.383, \mathrm{p}<0.05)$. Con respecto al BPE la correlación fue $\mathrm{rs}=0.383$, $\mathrm{p}<0.05$, lo que indica una baja correlación. Por otro lado, el consumo alimenticio de plantas durante la época seca y lluviosa no mostró diferencias significativas $(\mathrm{Z}=1.608, \mathrm{p}=0.949)$ (Fig. 1).

La prueba de $\mathrm{X}^{2}$ aplicada muestra que el consumo alimenticio en función de la forma biológica de las plantas no es significativo $\left(\mathrm{X}^{2}=12, \mathrm{p}=0.213\right)$ aunque existe tendencia a consumir mayormente especies del estrato arbóreo (Fig. 2).

\section{DISCUSIÓN}

Los venados consumieron en total 28 especies: 15 del Bosque Mesófilo de Montaña
(BMM), nueve del Bosque de Pino Encino (BPE) y cuatro de las partes perturbadas del BPE. Se apreciaron tres grados de preferencias de consumo: tres especies de alto consumo mayores al $11 \%$, siete de consumo moderado mayores al $2 \%$ y 14 de consumo menor o igual al 2\% (Cuadro 1). De las nueve especies consumidas por los venados en el BPE cuatro fueron consumidas moderadamente y seis fueron de bajo consumo. Una de las predicciones del presente estudio propone que podría ocurrir un consumo mayor en el BMM con respecto al BPE. EL 79.44\% del total consumido se realizó hacia 15 especies del BMM, mientras que tan sólo el $20.56 \%$ fueron producto del consumo de nueve especies del BPE con lo cual, aunque no se pudo comprobar estadísticamente la primera hipótesis, los datos son indicativos de que ésta puede ser correcta. Se considera que la preferencia de consumo de plantas del BMM por parte de los venados radica en la más amplia disponibilidad (en espacio y tiempo) de alimento a lo largo del año. Así mismo, el $\mathrm{BMM}$ provee a estos cérvidos de una mayor 


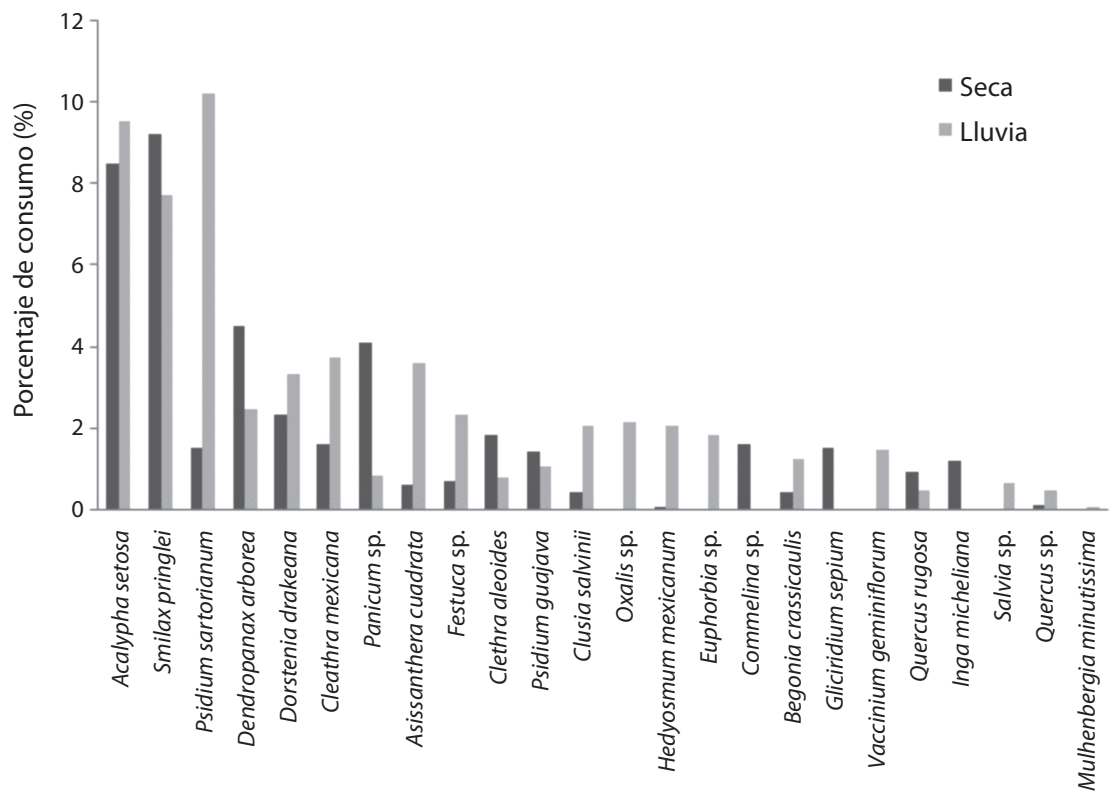

Especies

Fig. 1. Comparación del consumo de especies de plantas por el venado cola blanca durante la época seca y lluviosa. Fig. 1. Comparison of plant consumption by white-tailed deer during the dry and rainy seasons.

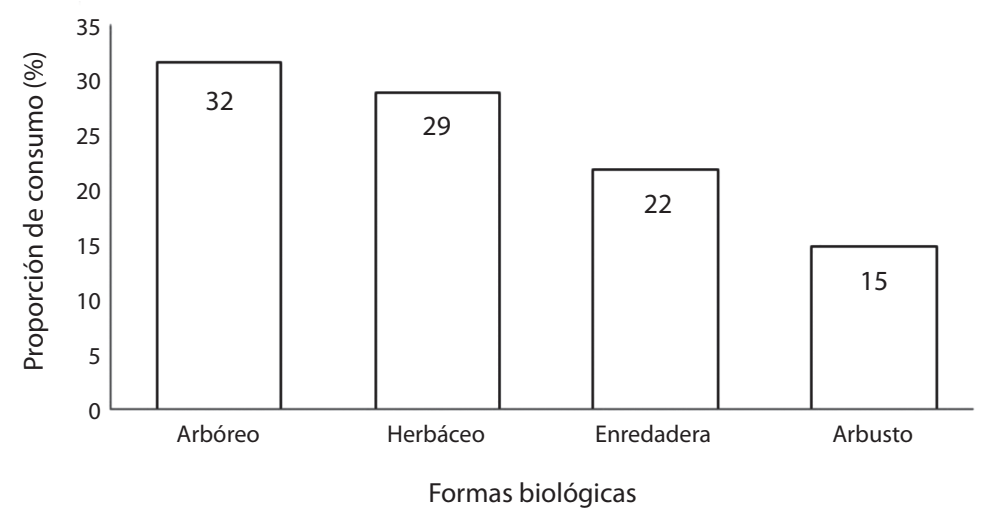

Fig. 2. Proporción de especies consumidas por el venado cola blanca con base en la forma biológica de crecimiento de las plantas.

Fig. 2. Proportion of species consumed by white-tailed deer based on the biological form of plant growth.

cobertura, lo cual es otra razón por la que consumieron plantas de este tipo de vegetación, pues les es posible reducir los riesgos de depredación, sobre todo después del ramoneo cuando se echan y rumian (Villarreal \& Gabriel 1986). Posiblemente este sea de los primeros estudios efectuado sobre hábitos alimentarios de esta especie en un BMM.

Cuatro especies fueron consumidas por los venados en las zonas perturbadas del BPE, entre estas plantas, cabe destacar a Pteridium aquilinum, un helecho indicador de 
perturbación y que está presente en algunas zonas del área de estudio. Al respecto, varios autores comentan la necesidad de algunos herbívoros de consumir especies que les provean de nutrientes o energía a pesar de poseer compuestos secundarios, como en el caso de este helecho, para llevar a cabo sus funciones vitales (Church 1988, McArthur et al. 1991, Ernest 1994, Provenza 1995, Silva-Villalobos et al. 1999, Colín-Negrete \& Bernal-Barragán 2005). Durante la temporada seca disminuye sustancialmente la disponibilidad de alimento y posiblemente los venados tiendan a consumir helechos como el que se menciona. Esto apoya lo citado por otros autores toda vez que $P$. aquilinum fue consumida sólo durante la época seca (Stanley-Smith 1992, Aguilar-Ortigoza 1994, Zepeda-Gómez \& Velázquez-Montes 1998).

La baja relación entre los porcentajes de consumo y los VI que consumieron los venados en el BMM, indica que los venados están siendo selectivos, vgr: 12 de las 14 especies fueron seleccionadas para su consumo aun cuando estas plantas obtuvieron VI bajos. En particular destacan dos especies como altamente seleccionadas: A. setosa y $S$. pringlei; sin embargo, en el muestreo realizado no se encontró a $S$. pringlei, por lo que no se obtuvo su VI, por lo que sólo se puede afirmar que hubo una fuerte selectividad hacia $A$. setosa.

Con respecto a la correlación aplicada a los porcentajes de consumo y los VI de las especies de plantas en el BPE, se obtuvo un valor que indica una correlación alta, es decir, los venados consumen estas especies por la frecuencia y oportunidad en esta época de lluvias y porque pueden tener altos valores nutrimentales.

Comparando las dos épocas, se puede observar que $A$. setosa fue consumida tanto en época de lluvias como en seca, por lo que pudiera tratarse de una especie clave de consumo, posiblemente por la calidad y cantidad de nutrientes que esta especie posee a pesar de no tener un VI alto (5\%). Esto muestra la necesidad de efectuar estudios comparativos de tipo bromatológico de esta especie con otras en el área de estudio. Algunos autores mencionan que los herbívoros poseen una desarrollada capacidad para detectar los nutrientes que las plantas poseen, a través del olfato y el sabor (Church 1988, Provenza 1995, Plata et al. 2009). De probarse el mayor valor nutritivo de $A$. Setosa, en programas de mejoras de hábitat para el manejo de fauna silvestre como el venado, esta especie sería una de las que tendrían que favorecerse (Gallina et al. 1981, Villarreal \& Gabriel1986, Gallina 1993, Sánchez-Carrillo 2011).

Por otra parte, las especies C. procumbens y $D$. arborea que se consumieron en alto porcentaje durante la época de lluvias, junto con $D$. drakeana, serían otras especies que debieran de tomarse en cuenta, pues también fueron fuertemente preferidas por los venados para su consumo.

El consumo de plantas en el estrato arbóreo fue el mayormente consumido con $31.79 \%$, mientras que el arbustivo fue el de menor consumo con $15.37 \%$. La prueba de $\mathrm{X}^{2}$ aplicada a la variación de los hábitos alimentarios de los venados cola blanca a lo largo del año en función de la forma biológica no fue significativa, sin embargo durante el mes de abril, consumieron mayormente árboles. Esto se debe a que disminuye substancialmente la disponibilidad de alimento del estrato herbáceo, dado que son los últimos meses de estiaje y por lo tanto, los venados dirigen su atención al estrato arbóreo. Durante el mes de noviembre consumieron mayormente hierbas lo que resulta razonable si tomamos en cuenta que la abundancia y frecuencia de esta forma biológica estuvo más disponible.

Con base en la variación de la alimentación entre la época seca y la época de lluvia, el resultado de la prueba estadística no fue significativo; esto quiere decir que no existe una tendencia a preferir algún estrato biológico en determinada época del año. En este sentido se comportan de manera similar a como lo refieren otros autores en estudios realizados en diferentes regiones de México y Centroamérica donde predominan los bosques templados y tropicales (Narváez et al. 1983, Clemente 1984, Medina-Flores 1986, Di Mare 1994, Silva-Villalobos 1999, Arceo et al. 2005, Villarreal-Espino-Barros et al. 2008, 
Hernández-Silva et al. 2011, Sánchez-Carrillo et al. 2011, González \& Briones-Salas 2012).

Del presente estudio se concluye que los venados prefirieron consumir especies vegetales del Bosque Mesófilo de Montaña (79.44\%) con respecto a las del Bosque de Pino-encino (20.56\%). Los datos permiten establecer cierta tendencia demostrable en la selectividad de 12 de las 14 especies de plantas localizadas en el BMM, mientras que para el BPE no se aprecia tal tendencia. Además, las especies clave que forman parte de la alimentación elemental de los venados fueron: Acalypha setosa, Smilax pringlei, Psidium sartorianum y Dendropanax arborea.

\section{AGRADECIMIENTOS}

Al Personal de la Comisión Estatal de Parques Naturales y de la Fauna del Gobierno del Estado de México por las facilidades otorgadas para la realización de la presente investigación. A Carmen Zepeda Gómez, por el apoyo en la determinación de las especies de plantas. A Oscar Sánchez, Guillermina Flores, Victoria Rivas Manzano por sus valiosas aportaciones al manuscrito.

\section{RESUMEN}

El venado cola blanca es una especie con una gran plasticidad conductual y de adaptación en diferentes hábitat. En el Parque Sierra Nanchititla en el Estado de México se realizó un estudio para determinar los hábitos alimentarios en el bosque mesófilo de montaña (BMM) y bosque de pino-encino (BPE). De junio 1990 a mayo 1992 se recolectaron 104 muestras de excremento de venado en las dos zonas de estudio. Aplicamos el índice de Morisita y la correlación de Spearman para determinar la preferencia alimentaria. Se utilizó el análisis histológico de heces fecales para herbívoros las cuales se compararon con muestras de tejidos vegetales de plantas de la zona de estudio. Los resultados muestran que consume el $79.44 \%$ de especies vegetales del BMM y $20.56 \%$ del BPE. Existe cierta tendencia en la selectividad de 12 de las 14 especies de plantas localizadas en el BMM, mientras que para el BPE no se aprecia tal tendencia. Las especies clave que forman parte de la alimentación elemental del venado fueron: Acalypha setosa, Smilax pringlei, Psidium sartorianum y Dendropanax arborea. El consumo de plantas no varió significativamente entre la época seca y lluviosa en función de la forma biológica $\left(\mathrm{X}^{2}=12, \mathrm{p}=0.21\right)$. Sin embargo, durante la época seca existe cierta tendencia a consumir árboles y a finales de la época de lluvia a consumir hierbas $(Z=1.61$, $\mathrm{p}=0.95$ ). Los datos indican que el venado puede ser selectivo con plantas del BMM, mientras que para el BPE tiende a ser oportunista.

Palabras clave: venado cola blanca, hábitos alimentarios, bosque mesófilo de montaña, bosque de pino-encino, México.

\section{REFERENCIAS}

Aguilar-Ortigoza, C.A. 1994. La vegetación de la zona núcleo del parque Sierra de Nanchititla. Revista de la Escuela de Ciencias, Universidad Autónoma del Estado de México. México 4: 6-16.

Arceo, G., S. Mandujano \& S. Gallina. 2005. Diet diversity of white-tailed deer (Odocoileus virginianus) in a tropical dry forest in Mexico. Mammalia 69: 159-167.

Bonilla, G. 1991. Métodos prácticos de inferencia estadística. Trillas, México.

Brower, J.E. 1977. Field and laboratory methods for general ecology. Brown Publishers, Atlanta, EE.UU.

Camargo-Sanabria, A. \& S. Mandujano. 2011. Comparison of pellet-group counting methods to estimate population density of white-tailed deer in a Mexican tropical dry forest. Trop. Con. Sc. 4: 230-243.

Catana, H.J. 1963. The wandering quarter method of estimating population density. Ecology 44: 349-360.

Ceballos, G. \& G. Oliva. 2005. Los mamíferos silvestres de México. CONABIO y Fondo de Cultura Económica. México.

Church, D.C. 1988. The ruminant animal: digestive physiology and nutrition. Prentice Hall, Englewood Cliffs, Nueva Jersey, EE.UU.

Clemente, S.F. 1984. Utilización de la vegetación nativa en la alimentación del venado cola blanca (Odocoileus virginianus Hays) en el estado de Aguascalientes. Tesis de Maestría, Colegio de Posgraduados, Instituo de Enseñanza e Investigación en Ciencias Agrícolas, Chapingo, Estado de México, México.

Colín-Negrete, J. \& H. Bernal-Barragán. 2005. Reseña de "nutrición del venado cola blanca" De Roque Gonzalo Ramírez Solano. Ciencia UANL 07: 114-116.

Cottam, G. \& J.T. Curtis. 1957. The use of distance methods in phytosociological sampling. Ecology 37 : 451-460.

DiMare, M. 1994. Hábitos alimentarios del venado cola blanca en la Isla San Lucas; puntarenas, Costa Rica, p. 73-89. In C. Vaughan \& M. Rodríguez (eds.). Ecología y manejo del venado cola blanca en México y Costa Rica. EUNA, Heredia, Costa Rica. 
Ernest, K. 1994. Resistance of creosote bush to mammalian herbivory: temporal consistency and browsing-induced changes. Ecology 75: 1684-1692.

Fracker, S.B. \& J.A. Brischle. 1944. Measuring the local distribution of Ribes. Ecology 25: 283-303.

Gallina, S., M.E. Maury \& V. Serrano. 1981. Food habits of while-tailed deer, p. 133-148. In P.F. Folliott \& S. Gallina (eds.). Deer biology, habitat requirements, and management in Western North America. Instituto de Ecología, México, México.

Gallina, S. 1993. White-tailed deer and cattle diets at the Michila Durango, México. J. Range Manage. 46: 487-492.

Gallina, S. \& H.M. Maury. 1978. Hábitos Alimenticios del Venado Cola Blanca (Odocoileus virginianus refinesque) en la Reserva La "Michila" Edo. de Durango. Tesis de Licenciatura, Universidad Nacional Autónoma de México, México.

García, E. 1981. Modificaciones al sistema de clasificación de Köppen para adaptarlo a las condiciones de la República Mexicana. Instituto de Geografía, Universidad Nacional Autónoma de México, México.

González, G. \& M. Briones-Salas. 2012. Dieta de Odocoileus virginianus (Artiodactyla: Cervidae) En un bosque templado en el norte de Oaxaca, México. Rev. Biol. Trop. 60: 447-457.

Hall, R. 1981. The mammals of North America.Wiley, Nueva York, EE.UU.

Henke, S.E., S. Demaris \& J.A. Pfister. 1988. Digestive capacity and diets of white tailed deer and exotics ruminants. J. Wildl. Manag. 52: 595-598.

Hernández-Silva, D.A., E. Cortés-Díaz, J.L. ZaragozaRamírez, P.A. Martínez Hernández, G.T. González-Bonilla, B. Rodríguez-Castañeda \& D.A. Hernández-Sedas. 2011. Hábitat del venado cola blanca, en la Sierra de Huautla, Morelos, México. Acta Zool. Mex. 27: 47-66.

Holechek, J.L., M. Vavra \& R.O. Pieper. 1982. Methods for determining the nutritive quality of ruminant diets: A review. J. Anim. Sci. 54: 363-376.

Horn, H.S. 1966. Measurement of "overlap" in comparative ecological studies. Am. Nat. 100: 419-424.

INEGI. 1987. Síntesis geográfica nomenclator y anexo cartográfico del Estado de México. SPP. México.

Krebs, Ch.J. 1989. Ecological Methodology. Harper \& Row, Nueva York, EE.UU.

Mandujano, S. 2011. Consideraciones para el manejo el manejo del venado cola blanca en UMA extensivas en bosques tropicales, p. 249-275. In O. Sánchez, P. Zamorano, E. Peters \& H. Moya (eds.). Temas sobre conservación de vertebrados silvestres en México. SEMARNAT, México D.F., México.
McArthur, C., A.E. Hagerman \& C.T. Robbins. 1991. Physiological strategies of mammalian herbivore against plant defences, p. 103-114. In R. Palo \& C.T. Robbins (eds.). Plant defenses against herbivore. CRC, Boca Raton, Florida, EE.UU.

Martínez, M.A., V. Molina, F. González, J.S. Marroquín \& J. Novar. 1997. Observations of white-tailed deer and cattle diets in México. J. Range. Manage. 50: 253-257.

McCullough, D.H. 1985. Variables influencing food habits of white-tailed deer on the George Reserve. J. Mamm. 68: 323-329.

Medina-Flores, J.A. 1986. Programa de conservación y aprovechamiento cinegético del venado cola blanca en la "Sierra Fría", Aguascalientes, p. 62-110. In I Simposio sobre el venado en México. Facultad de Medicina Veterinaria y Zootecnia. Universidad Nacional Autónoma de México, México.

Morales-García, O. 1985. Análisis cuantitativo de las dietas de ganado vacuno y venado cola blanca en La Michila, Durango. Tesis de Licenciatura, Universidad Nacional Autónoma de México, México.

Morisita, M. 1959. Measuring of interspecific association and similarity between comunities. Mem. Fac. Sci. Kyushu Univ. Ser. E, Japón.

Narváez, R.T., T. Rivera \& E. Gizzi. 1983. El venado cola blanca sus hábitos alimentarios. Dirección General de la Fauna Silvestre, México.

Ortega, A., S. Mandujano, J. Villarreal, M.I. Dimari, H. López-Arevalo, M. Correa \& M. Molina. 2011. White-tailed deer in Latin America, p. 565-597. In D. Hewitt (ed.). Biology and Management of WhiteTailed Deer, CRC, Taylor \& Francis Group, Boca Raton, Florida, EE.UU.

Peña, N.J. \& R. Habib. 1980. La Técnica Microhistológica: Un método para determinar la composición botánica de la dieta de Herbívoros. Serie Técnico-científica III-1. Departamento de manejo de pastizales. RELCINIP-SARH. Chihuahua, México.

Plata, F.X., S. Ebergeny, J.L. Resendiz, O. Villarreal, R. Bárcenad, J.A. Viccone \& G.D. Mendoza. 2009. Palatabilidad y composición química de alimentos consumidos en cautiverio por el venado cola blanca de Yucatán (Odocoileus virginianus yucatanensis). Arch. Med. Vet. 41: 123-129.

Provenza, F.D., J.A. Pfister \& C.D. Cheney. 1992. Behavioral toxicology of Iivestock ingesting plant toxins. J. Range. Manage. 45: 30-36.

Provenza, F. 1995. Postingestive feedback as an elementary determinant of food preference and intake in ruminants. J. Range. Manage. 48: 2-17.

Quintanilla, G.J., J. Reyna, R.G. Ramírez \& J. Aranda. 1988. Determinación de la composición botánica de la dieta del venado cola blanca (Odocoileus 
virginianus texanus) en los agostaderos del norte de Nuevo León, p. 50-61. In 11 Simposio sobre el venado en México. Facultad de Medicina Veterinaria y Zootecnia. Universdidad Nacional Autónoma de México, México.

Sánchez-Carrillo, B. 2011. Plan de manejo del venado cola blanca (Odocoileus virginianus mexicanus), en la comunidad de Aguacatitla, Hidalgo. Tesis de licenciatura, Universidad Autónoma Chapingo, México.

Silva-Villalobos, G., S. Mandujano, G. Arceo, S. Gallina \& L.A. Pérez-Jiménez. 1999. Nutritional value of plants consumed by the white-tailed deer in a tropical forest of Mexico. V. Sil. Neotrop. 8: 1-6.

Smith, W.P. 1991. Odocoileus virginianus. Mammal. Species 388: 1-13.

Stanley-Smith, G. 1992. Toxification and detoxification of plant compouds by rumiants: an overview. J. Range. Manage. 45: 25-30.

Vangilder, L.D., O. Torgerson \& W.R. Porath. 1982. Factors influencing diet selection by white tailed deer. J. Wildl. Manage. 46: 711-718.

Vaughan, C. \& A. Rodríguez. 1995. Conservación del venado cola blanca en el neotropico, p. 25-32. In
Ecología y manejo del Venado cola blanca en México y Costa Rica. Serie de Conservación Biológica y desarrollo Sostenible. Universidad Nacional, Costa Rica.

Villarreal, G. \& J. Gabriel. 1986. Importancia cinegética y comportamiento del venado cola blanca (Odocoileus virginianus texanus) en el noreste de México, p. 11-138. In I Simposio sobre el venado en México. Facultad de Medicina Veterinaria y Zootecnia. Universidad Nacional Autónoma de México, México.

Villarreal-Espino-Barros, O.A., L.E. Campos-Armendia, T.A. Castillo-Martínez, I. Cortes-Mena, F.X. PlataPérez \& G.D. Mendoza-Martínez. 2008. Composición botánica de la dieta del venado temazate rojo (Mazama temama), en la Sierra Nororiental del Estado de Puebla. Universidad y Ciencia 24: 183-188.

Zar, J.H. 1984. Biostatistical Analysis. Prentice-Hall, Englewood Cliffs, Nueva Jersey, EE.UU.

Zepeda-Gómez, C. \& E. Velázquez-Montes. 1999. El bosque tropical caducifolio de la Vertiente sur de la Sierra de Nanchititla, Estado de México: La composición y la afinidad geográfica de su flora. A. Bot. Mex. 46: 29-55. 
